\title{
Reactive-Transport modeling as a technique for understanding coupled biogeochemical processes in surface and subsurface environments
}

\author{
P. Regnier ${ }^{1, \star}$, P. Jourabchi ${ }^{1}$ \& C.P. Slomp ${ }^{1}$ \\ 1 Biogeochemical Systems Dynamics, Dept. of Geochemistry, Faculty of Earth Sciences, \\ Utrecht University, P.O. Box 80021, 3508 TA Utrecht, the Netherlands \\ * Corresponding author: p.regnier@geo.uu.nl
}

Manuscript received: March 2002; Accepted: January 2003

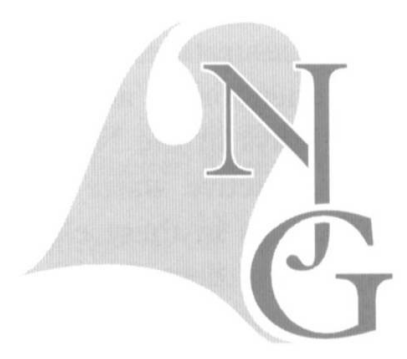

\begin{abstract}
Reactive-transport models contribute significantly to the field of modern geosciences. A general mathematical approach to solving models of complex biogeochemical systems is introduced. It is argued that even though mathematical models for reactive-transport simulations can be developed at various levels of approximation, the approach for their construction and application to the various compartments of the hydrosphere is fundamentally the same. The workings of coupled transport-reaction systems are described in more detail by means of examples, which demonstrate the similarities in the approach. Three models of the carbon dynamics in redox-stratified environments are compared: porous media flow problems in a coastal sediment and in a contaminated groundwater system; and a surface flow problem in a eutrophic estuary. Considering the interdisciplinary nature of such models, a Knowledge Base System for biogeochemical processes is proposed. Incorporation of the proposed knowledge base in an appropriate modeling framework, such as the Biogeochemical Reaction Network Simulator, proves an effective approach to the modeling of complex natural systems. This methodology allows for construction of multicomponent reactive-transport models applicable to a wide range of problems of interest to the geoscientist.
\end{abstract}

Keywords: Reactive-Transport modeling, Redox dynamics, Knowledge Base of biogeochemical processes, Estuaries, Early diagenesis

\section{Introduction}

All biogeochemical transformations on the Earth surface are the result of interaction processes in open systems. Many natural processes such as rock weathering and soil formation, the transfer of constituents from land to sea, the dissolution/precipitation of minerals within the ocean, the early diagenetic processes in unconsolidated sediments, and the metamorphic transformation of rocks can be generally described by the coupling between fluid flow, constituent transport, and biogeochemical interactions.

Reactive-transport modeling is an emerging research field, which aims at a comprehensive, quantitative, and ultimately predictive treatment of chemical transformations and mass transfers within the earth system. Reactive-transport models (RTMs) provide platforms for testing concepts and hypotheses, and for integrating new experimental, observational, and theoretical knowledge about geochemical, biological and transport processes. Through numerical computation and simulation, RTMs provide most valuable diagnostic and prognostic tools available for elucidating the inherently complex dynamics of natural and engineered environments. Furthermore, they bridge the gap between fundamental, process-oriented research and applied research in the fields of operational modeling, environmental engineering and global change. Examples of application areas relevant to the North Sea region are nutrient dynamics in river 
drainage basins and estuaries, eutrophication in coastal systems, contaminant transport in aquifers, and hydrocarbon migration and maturation in sedimentary basins.

RTMs are traditionally developed and used to investigate the fate and transport of a selected set of chemical constituents within a given compartment of the earth system, mainly at the local or regional scale. The modeling process involves four fundamental steps (Boudreau, 1997):

- problem identification;

- model formulation;

- solution of the model;

- interpretation of the results.

In this paper, we mainly focus on the first two steps. Problem identification is critical as the model formulation cannot be carried out without prior definition of its subject and objective. Problems can be identified in very general terms with respect to the types of questions they pose. Question types can be in nature: 1) diagnostic (process-oriented); 2) metagnostic (i.e. system-oriented); and/or 3) prognostic (i.e. forecastoriented). Models are developed to answer these specific question(s). More precisely, they can be used to: combine knowledge acquired in various disciplines and/or environments; assess the relative importance of the various forcing mechanisms on the system behavior; test the sensitivity of an environmental system to changes in any of these forcing processes; investigate dynamics over spatial and/or temporal scales which are not readily accessible to observations; extrapolate results obtained in a specific environment to another situation; and, finally, to make predictions.

A common practice is the use of computer simulations based on mathematical models for the sole purpose of data verification. However, the true value of mathematical models is in improving our understanding of the environmental system under study. This level of understanding is often greater than that achieved through data alone. For example, many biogeochemical studies are still restricted to a description of the environmental system status, based on sparse experimental data. These studies often involve the determination of concentrations within various liquid and solid phases along a spatio-temporal support, with little emphasis on the biogeochemical rates responsible for the distribution between these phases. The understanding of the system behavior gained on the basis of such experimental data is thus limited to an instantaneous and qualitative picture of the environment under investigation.

Another important aspect of environmental studies is the complex interplay between competing processes present in the system. For example, measured concen- trations are influenced by competitive reaction-transport processes, whose effects on the ecosystem must be evaluated collectively. Such an evaluation can hardly be achieved with field and experimental data alone. It is becoming increasingly clear that in order to understand the spatial and temporal heterogeneities of the environment and to provide a quantitative basis for its study, the simultaneous integration of the major regulatory processes responsible for the biogeochemical behavior of reactive species is needed. A modeling approach, which is capable of integrating transport and transformation processes characterized by a wide range of time and space scales, is particularly appropriate in these studies.

It is only over the last decade that a relatively rigorous quantitative treatment of biogeochemical interactions in natural systems has been performed. This is partly due to the fact that complex problems often require significant computational power. However, a more essential reason is that various disciplines involved in such studies have largely evolved independently. The aim of this paper is to provide a general mathematical approach to solving models of complex biogeochemical systems. Considering the interdisciplinary nature of such models, a Knowledge Base system for biogeochemical processes is proposed. The usefulness of the Knowledge Base approach is demonstrated through three examples of carbon dynamics in redox-stratified environments.

\section{Mathematical models}

\section{Formulation}

Briefly, the model formulation involves two fundamental steps: (1) selection of an environmental system, defined as a subunit of the environment separated by a boundary from the rest of the world; (2) selection of the complexity of the 'internal' system description (Schwartzenbach et al., 1993). The resulting model structure will be determined from the trade-off between the selected levels of spatial, temporal and dynamical complexity. Nihoul (1975, 1993) defines the structure of a mathematical model by the following characteristics:

- Span: dimensions in physical (support) and state (scope) spaces. A reduction of the size of the system can be carried out, both in term of scope (aggregation) and support (averaging in space and time). The spatio-temporal averaging process defines the scales that the model can represent: its spectral window.

- Set of operant state variables: the operant state variables are the mean values associated to a 
specific spectral window. These spectral windows correspond to the scales of external forcing or intrinsic mechanisms.

- Evolution equations: the evolution equations are a set of conservation laws, which describe the spatiotemporal evolution of the operant state variables. The evolution equations applicable to the set of operant variables (physical, chemical, biological) are all particular expressions of a unique basic equation.

- Purview: spread in physical space (external scale), both in terms of 'duration' and 'reach'. The delineation of a model defines the interaction of the system with the outside world, and in particular, its boundary conditions.

- Resolution: definition (mesh size or internal scale), precision and realism.

- Accuracy: ability to reproduce reality in the framework of the subject, objectives, and the means.

It is worthwhile to note some of the important implications of the above model characteristics. These characteristics highlight the fact that describing the real world environment through a numerical model requires much more than the definition of a set of evolution equations. It also demonstrates that a wide range of models can be produced to describe the same environment depending on the various levels of spatial, temporal, and dynamical approximations used. While most of the model characteristics are system specific, the fundamentals of evolution equations are common to all reactive-transport model formulations. The following section is a general overview of conservation laws for defining the evolution equations.

\section{The evolution equations}

\section{The fundamental conservation law}

The fluid constitutes a continuous medium whose static and dynamic properties are determined by an equation of state and fundamental conservation laws (mass, momentum, energy). If a requirement of mass conservation for each independent chemical species is included, a full set of nonlinear partial differential equations governing the hydrologic, thermal, and chemical evolution of a natural system is obtained (Lasaga, 1997).

The equations describing the spatial and temporal evolution of all state variables may in fact be derived from a unique conservation equation, which states that the local change in time of a state variable $y$ (defined per unit mass) within an elementary volume, $V$, of fluid results from the production and/or destruction of $y$ inside $V$, and a transport imbalance through the surface bounding $V$ (Nihoul, 1993). In the case of an inviscid, perfect fluid, i.e. a fluid not submitted to any molecular, thermal agitation or internal shear stress, the conservation equation describing the physical, chemical, or biological evolution of an inviscid fluid obeys

$\frac{\partial}{\partial t}(\rho y)+\vec{\nabla} \cdot(\rho y \vec{u})=\rho P^{y}$

where $\rho\left[\mathrm{kg} \mathrm{m}^{-3}\right]$ is the fluid density; $P^{y}$ is the rate of production/destruction of $y ; \vec{u}\left[\mathrm{~m} \mathrm{~s}^{-1}\right]$ is the fluid velocity vector and $t$ is time $[\mathrm{s}]$.

\section{Conservation of mass and momentum}

A condition for mass conservation can be derived if $y$ $=1$ (in this case, $\rho y$ is the specific mass). In natural fluid systems, mass is neither destroyed nor created $\left(P^{y}=0\right)$. Furthermore, if the fluid is incompressible, which is often a reasonable assumption in hydrodynamics, $\rho$ is constant and equation ( 1 ) reduces to

\section{$\vec{\nabla} \cdot \vec{u}=0$}

An equation of motion can also be derived from equation (1) if $y=u_{n}$ (a component of the velocity vector) and from the fundamental relationship which states that the rate of change of momentum in a physical system is equal to the sum of all forces acting on it.

If we consider that a water particle on the Earth is subject to the forces of acceleration due to gravity, the effects of Earth's rotation, or internal pressure gradients, we obtain the sum of forces, $F_{p e f}$ acting on a perfect fluid moving in a rotating reference frame:

$$
F_{\text {perf }}=\rho \vec{g}-2 \rho \vec{\Omega} \times \vec{u}-\vec{\nabla} p
$$

where $\vec{g}\left[\mathrm{~m} \cdot \mathrm{s}^{-2}\right]$ is the gravitational acceleration, $p$ $\left[\mathrm{N} \cdot \mathrm{m}^{-2}\right]$ the pressure of the fluid, and $\vec{\Omega}\left[\mathrm{rad} . \mathrm{s}^{-1}\right]$ the angular velocity from the rotation of the Earth.

In order to describe the motion of viscous fluids, an additional contribution $F_{v i s c}$ from the forces associated with internal friction must be considered. In laminar flow, the viscous effects of a fluid are related to its internal properties defined as molecular viscosity, $\mu$ $\left[\mathrm{m}^{2} \cdot \mathrm{s}^{-1}\right]$, (Officer, 1976) and are expressed as

$$
F_{v i s c}=\rho \vec{\nabla}(\mu \vec{\nabla} \cdot \vec{u})
$$

Combining equations (1), (3) and (4) leads to the conservation law of momentum:

$\rho \frac{\partial \vec{u}}{\partial t}+\rho \vec{u} \vec{\nabla} \cdot \vec{u}=\rho \vec{g}-2 \rho \vec{\Omega} \times \vec{u}-\vec{\nabla} p+\rho \vec{\nabla}(\mu \vec{\nabla} \cdot \vec{u})$ 
Equation (5) is a reduced form of the NavierStokes equations for viscous fluids. Because internal friction is expressed as a function of velocities alone, the vector equation (5) describing the dynamics of a homogeneous body of water of known density contains 4 unknowns, namely $u_{x}, u_{y}, u_{z}$ and $p$ in rectangular coordinates. An additional requirement takes the form of the continuity equation (2), which guarantees mass conservation. The system (equation 2 and 5 ) forms a set of 4 non-linear partial differential equations. These equations along with appropriate boundary conditions are sufficient for describing the temporal and spatial evolution of the instantaneous flow fields.

\section{The conservation equation for a constituent}

A conservation equation for any constituent $j$ with concentration $c_{j}$ [moles. $\mathrm{kg}^{-1}$ ] can also be obtained from equation (1). The flux in viscous fluids is made of an advective contribution associated with the fluid motion, but also of a diffusive contribution due to the molecular, thermal agitation of the fluid. This diffusive flux leads to a mass transfer in the same way as the shear stress of viscous fluid leads to a momentum flux between neighboring parcels of water. In this case, however, the force leading to a mass flux results from a gradient in chemical potential, instead of a velocity gradient (Boudreau, 1997). If $\eta\left[\mathrm{m}^{2} \cdot \mathrm{s}^{-1}\right]$ denotes the molecular diffusivity of $y$, the continuity equation for $c_{j}$ takes the general form

$$
\rho \frac{\partial c_{j}}{\partial t}+\rho \vec{u} \cdot \vec{\nabla}_{c_{j}}=\rho P^{c_{j}}+\rho \vec{\nabla} \cdot\left(\eta \vec{\nabla} c_{j}\right)
$$

Equation (6) states that a local change in concentration, $c_{j}$, is due to either a local transformation process $P^{\prime}$, or a divergence in advective or diffusive fluxes. This equation forms the cornerstone of reactivetransport models and will be further discussed in the following sections.

\section{Temporal, spatial and dynamical approximation}

By definition, mathematical models are intimately related to the concept of approximation. In every model, the approximations at the spatial, temporal, and dynamical levels are determined by: problem identification (questions addressed by the modeling process); the intensities of the external forcing mechanisms; and the intrinsic physical characteristics of the environmental system under investigation. Technical considerations such as computer power may impose an additional constraint on the degree of ap- proximation adopted during the development of the models.

In fluid dynamics, it is assumed that the fundamental equation describing fluid flows is known theoretically. However, it is not practically possible to describe the evolution of this physical system in its full complexity (Hirsch, 1988). The modeling of this physical system requires approximations, which in turn introduce a certain level of model parameterization. In this case, the quantification of the control parameters can be performed in light of theoretical arguments. In contrast, biogeochemical dynamics cannot be defined entirely by fundamental evolution equations. For this reason, a large set of interaction processes are approximated through phenomenological laws. Consequently, we are unable to formulate complex biogeochemical systems as a set of fully general conservation laws derived from first principles. The following describes some aspects of the temporal, spatial, and dynamical approximations relevant to reactive-transport models.

\section{Temporal averaging and turbulence}

The continuity and momentum equations provide a theoretical framework for a quantitative description of the evolution of a homogeneous water mass. The basic hydrodynamic equations ( 2 and 5 ) govern the spatial and temporal distribution of the instantaneous flow fields. However, the detailed motion of turbulent flow is fundamentally unpredictable, because of the nonlinear nature of the Navier-Stokes equations (Stewart, 1975). As a result, the only variables that can be predicted with confidence are average quantities, i.e. statistical properties of the flow.

In order to provide a formal relationship between nonlinear terms and the internal stresses associated with turbulent motion, any state variable (e.g. the horizontal component of the velocity vector, $u_{x}$ ) appearing in the equations is defined as the sum of an average value $\left(\left\langle u_{x}\right\rangle\right)$ and a fluctuating part $\left(u_{x}{ }^{\prime}\right)$. Applying such an averaging operator to the momentum equation (5), one can readily show that the linear terms in the equation defining the average motion remain unchanged, but that additional contributions from nonlinear interactions are introduced. The products of the fluctuations arising from nonlinear interactions (the Reynolds stresses) are parameterized by means of the gradient of the averaged scalar quantities:

$$
\begin{aligned}
& -\cdot\left\langle u_{x}^{\prime} u_{x}^{\prime}\right\rangle=v_{x} \frac{\left\langle\partial u_{x}\right\rangle}{\partial x} ; \quad-\left\langle u_{x}^{\prime} u_{y}^{\prime}\right\rangle=v_{y} \frac{\left\langle\partial u_{x}\right\rangle}{\partial y} ; \\
& -\left\langle u_{x}^{\prime} u_{z}^{\prime}\right\rangle=v_{z} \frac{\left\langle\partial u_{x}\right\rangle}{\partial z}
\end{aligned}
$$


where $v\left[\mathrm{~m}^{2} \cdot \mathrm{s}^{-1}\right]$ is a coefficient of eddy viscosity, whose value is several orders of magnitude larger than typical values for molecular viscosities. Molecular effects can thus be included in the turbulent terms, such that the equation describing the average motion of an incompressible fluid subjected to turbulent mixing is in vector form

$\rho \frac{\langle\partial \vec{u}\rangle}{\partial t}+\rho\langle\vec{u}\rangle \vec{\nabla} \cdot\langle\vec{u}\rangle=$

$$
\rho \vec{g}-2 \rho \vec{\Omega} \times\langle\vec{u}\rangle-\vec{\nabla} p+\rho \vec{\nabla}(v \vec{\nabla} \cdot\langle\vec{u}\rangle)
$$

where all state variables refer to averaged quantities.

Reynolds stresses also appear in the inertial terms of equation (6) for any substance $c_{j}$. By analogy with the time-averaged momentum equation, these turbulent contributions can also be parameterized by means of the gradients of the averaged scalar fields. A new coefficient of eddy diffusivity, $\lambda$, is introduced such that the mean spatial and temporal evolution of a concentration field of a substance obeys

$$
\frac{\partial\left\langle c_{j}\right\rangle}{\partial t}+\langle\vec{u}\rangle \cdot \vec{\nabla}\left\langle c_{j}\right\rangle=P^{c_{j}}+\vec{\nabla} \cdot\left(\lambda \vec{\nabla}\left\langle c_{j}\right\rangle\right)
$$

In the above equation, $\lambda\left[\mathrm{m}^{2} \cdot \mathrm{s}^{-1}\right]$ is the coefficient of eddy diffusivity associated with the time-averaging process of the instantaneous equations. In general, turbulent fluxes are redefined as the dispersion of a fluid property, without any mass transfer arising from the average motion of the fluid. They can therefore be referred to as dispersive flow. In contrast, the advective flow refers to the mass transfer associated with this average motion.

The same averaging operator can be applied for time smoothing over much longer periods of time, leading to a new set of operant state variables (e.g. Regnier et al., 1998). In such an application of the averaging operator, larger scale fluctuations are not resolved, and the spectral window of the model (i.e. the time and space scales that the model can represent) is increased accordingly (Table 1).

This is in contrast to averaging the true 3D isotropic turbulence alone. In general, however, the mechanisms of motion associated with the fluctuating, unresolved processes will be parameterized according to a closure assumption similar to that given by equation (7). As a result, reactive-transport models defined by quite different spectral windows can be formalized through identical mathematical formulations.
Table 1. Hydrodynamic variability of the marine environment (taken from Nihoul \& Djenidi, 1991). The 'spectral windows' correspond to the scales of external forcings of intrinsic mechanisms.

\begin{tabular}{lll}
\hline Time scale & Spectral window & Small scale fluctuation \\
\hline 1 second & Microscale & Molecular diffusion \\
\hline 1 minute & Mesialscale & Eddy turbulence \\
\hline 1 hour & & 'Bliny turbulence' \\
\hline 1 day & Mesoscale & \\
\hline 1 week & Synopticscale & Mesoscale variability \\
\hline 1 month & Seasonalscale & 'Rossby turbulence' \\
\hline 1 year & Globalscale & Seasonal variability \\
\hline
\end{tabular}

\section{Reduction in support: spatial integration}

The dynamics of environmental systems can sometimes be approximated by integrating the conservation equations over one, or even two spatial dimensions. Consider the cross-sectional integration of the mass conservation equation of a solute constituent $c$ (equation 6). The average velocity of the flow through any section $A$ can be defined as (O'Kane, 1980):

$U(x, t)=\frac{1}{A(x, t)} \int_{A} u_{x} d A$

The total flow through $A$ is equal to $Q(x, t)=$ $U(x, t) A(x, t)$. The instantaneous mass flow rate $M$ through $A$ is then, by definition

$$
M(x, t)=\int_{A} u_{x} c d A
$$

Let $C(x, t)$ be the average of $c$ in $A(x, t)$, defined in the same manner as $U(x, t)$ in equation (10). If

$U^{\prime}=u_{x}-U$ and

$C^{\prime}=c-C$

define the deviations about the averages $U$ and $C$ at all points within $A$, the mass flow rate can be expanded according to:

$\int_{A} u_{x} c d A=A U C+\int_{A} U^{\prime} C^{\prime} d A$

Equation (13) shows that the instantaneous flux through $A(x, t)$ has been split into the sum of the flux due to the instantaneous average velocity $U(x, t)$, and a mass transport due to the correlation between ve- 
locities and concentrations within $A(x, t)$. In order to close the problem, i.e. to produce a single dependent variable $C$, the product of the spatial fluctuations is formulated as a linear function of the gradient of the average concentration,

$$
\int_{A(x, t)}\left(U^{\prime} C^{\prime}\right) d A=-K A \frac{\partial C}{\partial x}
$$

where $K\left[\mathrm{~m}^{2} \cdot \mathrm{s}^{-1}\right]$ is the dispersion coefficient associated with the spatial averaging process. This closure indicates that the cross-sectional fluctuations have been removed by averaging over space.

$A(x, t)$ and $U(x, t)$ can be calculated by the simultaneous solution of the cross-sectionally averaged continuity and momentum equations, which reads (neglecting possible density gradients along the $x$ direction):

$$
\begin{aligned}
& \frac{\partial A}{\partial t}=-\frac{\partial Q}{\partial x} \\
& \frac{\partial Q}{\partial t}+\frac{\partial}{\partial x}\left[\frac{Q Q}{A}\right]=-A g \frac{\partial \xi}{\partial x}+\frac{\partial}{\partial x}\left[\kappa \frac{\partial Q}{\partial x}\right]+\tau_{s}-\tau_{b}
\end{aligned}
$$

where $\xi[\mathrm{m}]$ is the water elevation; $\tau_{s}$ and $\tau_{b}\left[\mathrm{~m}^{3} \cdot \mathrm{s}^{-2}\right]$ are the surface tension at the surface and on the sidewalls of the system, respectively; and $\kappa\left[\mathrm{m}^{2} . \mathrm{s}^{-1}\right]$ is the coefficient of eddy viscosity arising from the product of the fluctuations of $U^{\prime}$ around the cross-sectional mean (Regnier at al., 1998).

Details about the cross-sectional integration of these equations are beyond the scope of this paper. Briefly, however, the integration over $A(x, t)$ has replaced the $x$-component of the velocity in the $3 \mathrm{D}$ equations with volume flux. The structure of the momentum equation is also modified in two ways (Nihoul, 1993). First, boundary momentum fluxes such as wind stress or bottom friction are introduced in the equations. Second, nonlinear contributions have given rise to products of the fluctuations around mean cross-sectionally averaged values.

\section{Dynamical approximations: Darcy's law for porous media}

There are many levels of quantification of the momentum conservation equations. The Navier-Stokes equation for incompressible viscous fluid (equation 8) is certainly one of the most fundamental macroscopic descriptions of fluid dynamics. In porous media, however, a phenomenological approach, which originates from Darcy, has been extensively used. Darcy's law provides a very good example of dynamical approxi- mation. A simple linear relationship between the fluid velocity, $U_{d}$, and the body forces acting on the fluid is proposed (Lasaga, 1997):

$U_{d}=-\frac{k}{\mu}(\vec{\nabla} p-\rho \vec{g})$

where $k\left[\mathrm{~m}^{2}\right]$ is the permeability tensor, $\mu$ the dynamic viscosity, $p$ is the fluid pressure, and $\vec{g}$ the gravity vector.

Equation (16) shows that in this phenomenological law, the two body forces susceptible to initiating fluid motion are gravity and pressure gradients. These forces are balanced by the drag force of the fluid medium introduced through the viscosity term, $\mu$. Darcy's law is widely used in groundwater modeling as well as in the field of early diagenesis (e.g. when sediments are subject to externally impressed flow).

Darcy's law can be derived directly from the Navier-Stokes equation (de Marsily, 1986). For this purpose, the assumptions that have to be made to reconcile both formulations are:

- The flow must be steady;

- The balance of forces is between gravity, pressure gradients, and friction;

- The velocity is linearly proportional to the forces.

\section{Coupled transport-reaction systems}

\section{The one-dimensional advection-dispersion-reaction $(A D R)$ equation}

Table 2 shows that geochemical reservoirs are characterized by widely differing time and space scales. Despite these variations in scale, similar continuum representations have often been used to describe the dynamic reactive transport in many compartments of the hydrosphere. For instance, the one-dimensional advection-dispersion-reaction (ADR) equation, obtained via spatial integration of the 3-D mass conservation equation, has been successfully applied to both surface flow

Table 2. Indicative characteristic time and space scales of some important geochemical reservoirs of the hydrosphere. Note that each of these systems exhibits variability over different spectral windows (see Table 1 for an example).

\begin{tabular}{lll}
\hline Geochemical reservoir & Time scale, $\mathbf{t}$ & Space scale, $\mathbf{L}$ \\
\hline $\begin{array}{l}\text { Rock weathering - soil } \\
\text { formation }\end{array}$ & $10^{4}-10^{5} \mathrm{yrs}$ & $\mathrm{L}_{\mathrm{z}} \sim 10^{-2}-10^{1} \mathrm{~m}$ \\
\hline Groundwater aquifer & $10^{0}-10^{2} \mathrm{yrs}$ & $\mathrm{L}_{\mathrm{x}} \sim 10^{1}-10^{5} \mathrm{~m}$ \\
\hline Sediment & $1-10^{3} \mathrm{yrs}$ & $\mathrm{L}_{\mathrm{z}} \sim 10^{-3}-10^{0} \mathrm{~m}$ \\
\hline Estuary & $10^{-2}-10^{-1} \mathrm{yrs}$ & $\mathrm{L}_{\mathrm{x}} \sim 10^{4}-10^{5} \mathrm{~m}$ \\
\hline
\end{tabular}


and porous media problems. Examples of surface flow problems are those pertaining to lakes, rivers, or estuaries, while porous media problems may involve sediments, groundwaters, or soils. In what follows, we discuss the $1 \mathrm{D}-\mathrm{ADR}$ equation applied to solute movement in surface waters and sediments, respectively.

\section{Surface waters}

A continuum representation of coupled mass transport and chemical reactions in surface waters can be described mathematically by a set of partial differential equations (PDEs) in time and space. Applying the cross-sectional integration of equation 6 to each of the $N_{t o r}$ species concentration, we obtain,

$$
\begin{aligned}
& \frac{\partial\left(A C_{j}\right)}{\partial t}+\frac{\partial\left(Q C_{j}\right)}{\partial x}= \\
& \frac{\partial}{\partial x}\left[K A \frac{\partial C_{j}}{\partial x}\right]+A P_{j} \quad\left(j=1, \ldots N_{t o t}\right)
\end{aligned}
$$

The above equation may be read as follows. At a point $x$ on the 1-D longitudinal curvilinear axis of the surface flow system, and at time $t, C_{j}(x, t)$ is the solute concentration measured as mass of solute per unit volume of water; $A(x, t)$ is the volume of water per unit length of the $x$ axis; $Q(x, t)$ is the volumetric flow rate of water, positive in value when flowing in the positive $x$ direction; $K(x, t)$ is the non-negative longitudinal dispersion coefficient with a dimension of length squared per unit time, and $P_{j}$ is the algebraic sum of all the production and destruction rates of $C_{j}$, given as a positive or negative concentration per unit time. Among the variables involved in Eq. (17), $A(x, t)$ and $Q(x, t)$ are variables that are provided by the solution of the spatially averaged continuity and momentum equations of the fluid (equation 15).

Equation (17) has infinitely many continuous solutions. Specifying physically appropriate boundary conditions, however, allows for the selection of a unique continuous solution. Such conditions are bounded by two extreme situations corresponding to no (Neumann condition) and infinite (Dirichlet condition) dispersion beyond the modeled domain. The set of nonlinear partial differential equations (17) along with their respective boundary conditions are used for the computation of fluxes of reactive species in continuous media. The flux consists of an advective contribution $\left[f_{a d v}=Q(x, t) C_{j}(x, t)\right]$ associated with the motion of the fluid itself and a dispersive contribution $\left[\mathfrak{f}_{\text {disp }}=A(x, t) K(x, t)\left(\partial C_{j}(x, t) / \partial x\right)\right]$. The eddy dispersion coefficient $K$, defined by equation (14), is a control parameter of the mathematical model.

\section{Sediments}

Early diagenetic processes describe the changes in concentrations with depth of a solid or porewater constituent in a sediment column. In modeling early diagenesis, the dynamics is generally resolved over the vertical direction, $z$, which denotes a position along the sediment column. The geometry of the system is assumed independent of both depth and time, i.e. $A(z, t)=$ constant. Under these conditions, the standard ADR equation for a solute species $C_{j}$ (mass per unit volume porewater) in sediments is (Berner, 1980)

$$
\frac{\partial C_{j}}{\partial t}+\omega \frac{\partial C_{j}}{\partial z}=\frac{\partial}{\partial z}\left[D_{T} \frac{\partial C_{j}}{\partial z}\right]+P_{j} \quad\left(j=1, \ldots N_{t o t}\right)
$$

Equation (18) may be read as follows. The concentration changes of a constituent $j$ at depth $z$ result from the dynamic balance between sedimentary advection, dispersive transfer, and biogeochemical transformations. In the absence of compaction or externally impressed flow, the advective flux in a $1 \mathrm{D}$ diagenetic model is essentially due to the burial rate, $\omega$, assumed to be constant here. It results from the sediment-water interface moving away from porewater as sediment accumulates, and is specified in unit length per unit time. The dispersion coefficient $D_{T}(z)$ is in general due to molecular diffusion (corrected for tortuosity), and bioturbation. The latter dispersion mechanism includes all displacements within the sediment produced by the activity of organisms (Boudreau, 1997).

Comparison of equations 17 and 18 for surface waters and sediments, respectively, show that the fundamental physical processes associated with advection and dispersion are not identical in the two models.

A similar conclusion would hold if a groundwater system had been considered. In surface waters, the advective flux arises from the movement of the mean flow $Q(x, t)$, and the eddy diffusion coefficient, $K$, is mainly due to the macroscopic averaging process over the spatial dimensions (cross-sectional integration). Molecular diffusion and true three-dimensional isotropic turbulence have a negligible contribution to $K$ in comparison to the mixing effects associated with the product of the fluctuations around the cross-sectional mean (equation 14). In sediments, however, it is the burial process, which drives the advective flux, and the diffusion coefficient, $D_{T}$, is essentially associated with molecular diffusion and biologically induced mixing (bioturbation). In fact, bioturbation is to sediment movement what turbulence is to flowing fluids, and this process can be treated similarly via the theory 
of Reynolds decomposition and averaging (Boudreau, 1997). A physical dispersion flux due to spatial deviations also exists in sediments, but this contribution is normally negligible, because flows are generally very small in these porous media (i.e. $<1 \mathrm{~cm} \cdot \mathrm{yr}^{-1}$ ).

The distinction between dispersion and advection strongly depends on the time and space scales of interest. However, despite the fact that the dispersive and advective processes may include quite different mechanisms of transport, it remains that the continuum representation of mass transport shows great mathematical similarities when applied to the different environments of interest to the geoscientist. A key objective of reactive-transport modeling is to develop numerical techniques that can take advantage of these similarities, and in turn, allow for the development of a common strategy for the modeling of complex biogeochemical reaction networks in various environments of the hydrosphere (e.g. surface waters, groundwater, sediments).

The production/destruction term, $P_{j}$, in equations 17 and 18 has not been discussed so far. This term can be written explicitly as the sum of all the individual reaction rates that affect the concentration of the $j^{\text {th }}$ species

$$
P_{j}=\sum_{m=1}^{N_{r}} v_{m, j} R_{m}
$$

where $R_{m}$ is the rate of a reaction of arbitrary form and $v_{m, j}$ is the number of moles of species $j$ involved in reaction $R_{m}$ (i.e., the stoichiometric coefficient of component $j$ in reaction $m$ ). Because $R_{m}$ is potentially a function of at least several concentrations of the system, (i.e. the rate laws are nonlinear) equations (17) and (18) each form a set of $N_{t o t}$ coupled partial differential equations that have to be solved simultaneously. Multispecies systems, therefore, require some special numerical treatment. These aspects are discussed in detail in Steefel \& Macquarrie (1996). In what follows, we propose a new and efficient mean to include and combine any set of biogeochemical reactions $\left(R_{m}\right.$ in equation 19) into Reactive-transport models. The modular approach presented here, based on the Knowledge Base concept, is essential if one aims at matching the progress in predictive environmental modeling to the rapidly increasing database on the rates and mechanisms of biogeochemical processes.

\section{The Knowledge Base of biogeochemical proces- ses}

Knowledge about key reaction processes in the biogeochemical dynamics of natural environments is continuously expanding. Field and laboratory based experiments are being conducted to identify reaction pathways, quantify reaction kinetics, and elucidate the interactions between biotic and abiotic processes. An important challenge, however, is to develop theoretical and computational tools that allow the systematic integration of new knowledge about the coupled biogeochemical processes, which together determine the cycling of elements in environmental systems. In this framework, a Biogeochemical Reaction Network Simulator (BRNS), based on the novel concept of an evolving 'Knowledge Base' (KB) has been developed (Regnier et al., 2002). In this approach, it is no longer the model itself, but rather an easily accessible, open resource element, the $\mathrm{KB}$, which contains the growing conceptual and quantitative understanding of individual biogeochemical pathways, and their interactions. It is through the $\mathrm{KB}$ that a continuous learning process and information exchange between experimentalists and modelers can be conducted. In addition to the $K B$, a newly developed automatic code generator and associated numerical engines constitute the BRNS.

The Biogeochemical Reaction Network Simulator (BRNS) provides a simulation environment in which transport processes are interfaced with relevant biogeochemical reactions. The BRNS consists of three key elements: 1) a MAPLE pre-processor containing an automated procedure for model code generation ('Automatic Code Generator'); 2) a numerical engine, combining standard routines for solving transport equations, and sets of coupled nonlinear process equations generated by the MAPLE pre-processor; and 3) a Web-distributed Knowledge Base (KB), currently under development. The Automatic Code Generator translates the information extracted from the Knowledge Base into Fortran code, which is then linked and compiled with the 'static' routines (e.g. transport algorithms; linear algebra solvers) (Regnier et al., 2002).

The logical structure of the Biogeochemical Reaction Network Simulator (Knowledge Base, MAPLE pre-processor and numerical engine) is shown in Fig. 1. A preliminary automated protocol to transfer information from the Knowledge Base (KB) to the preprocessor has been established. The protocol allows remote access to the complete list of biogeochemical reactions incorporated in the $\mathrm{KB}$ via the Internet. After the reactions of interest have been selected and their list submitted an automated procedure is started whereby the corresponding process files are extracted from the KB, and parsed into the MAPLE worksheet. The information from the files is compiled and the reaction matrix defining the reaction network is con- 


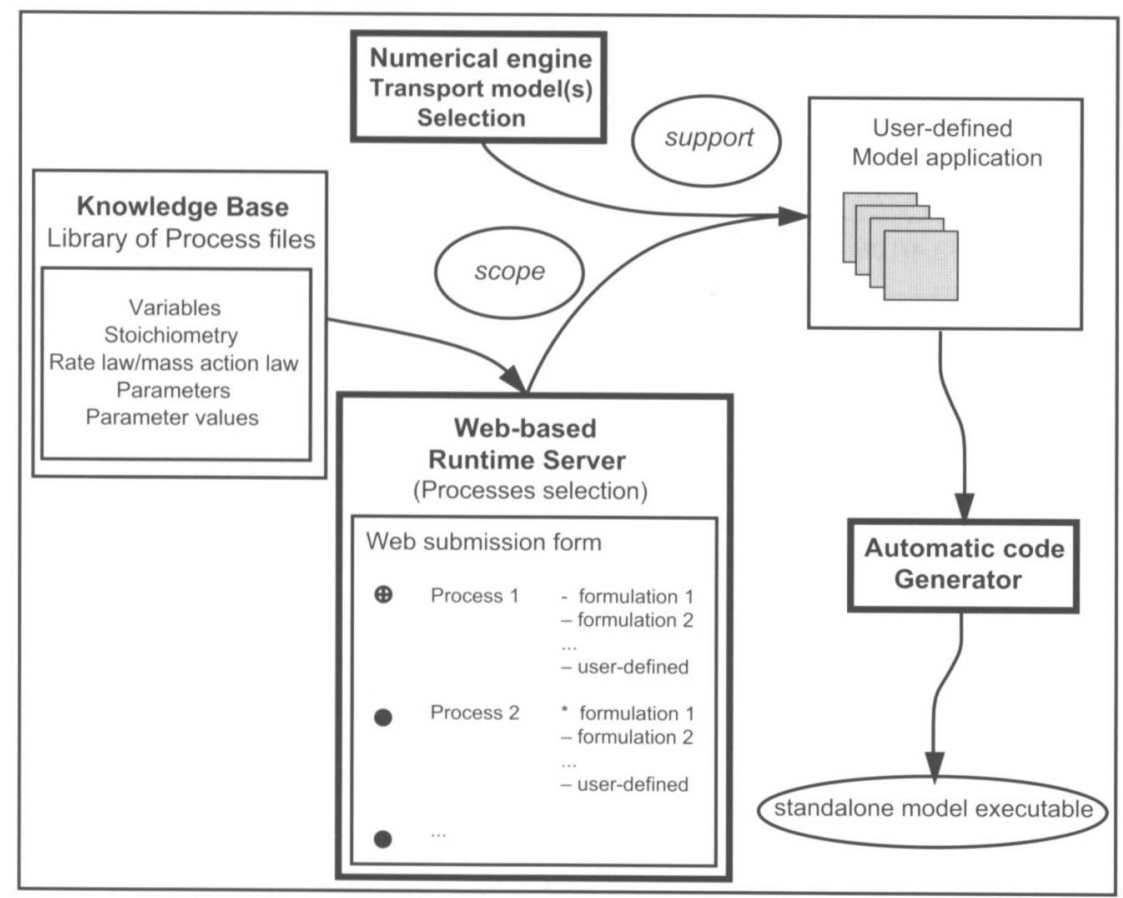

Fig. 1. Logical structure of the Biogeochemical Reaction Network Simulator.

structed. Consequently, the MAPLE preprocessor generates the FORTRAN code corresponding to the reaction network. The proposed automatic creation of the model executable is similar in approach to that of Ferris et al. (2000) in the area of computational mathematics, which has been successfully tested.

\section{The MAPLE pre-processor}

Reaction pathways are selected from the Knowledge Base to build a reaction network in which chemical species concentrations and/or microbial biomasses are coupled to one another via reaction rate and microbial growth rate terms. Because many of the biogeochemical processes of interest involve disparate time scales, stiff sets of differential equations are commonly produced that require the use of implicit solvers in order to be computationally efficient (Steefel \& MacQuarrie, 1996). The resulting equations describing the time dependent changes of species concentrations and biomasses are transformed into a set of linear equations using a Taylor series expansion. The Jacobian matrix, which includes partial derivatives of the reaction or growth rates with respect to the (unknown) concentrations and biomasses, completely defines the reaction system. A fully automated procedure based on the MAPLE symbolic programming language to calculate the derivatives of the functions (mass balance equations and, if equilibrium reactions are included, mass action equations) representing the reaction pathways is used. Both the functions and the Jacobian matrix are then automatically written out by the preprocessor in fully structured FORTRAN programming language. The MAPLE pre-processor allows one to construct reaction systems with arbitrary combinations of equilibrium and kinetic reactions which may take almost any form and thus offers an extremely flexible way to treat multi-component reaction systems (Chilikapati, 1995; Regnier et al., 1997). To perform reactivetransport calculations, the reaction network calculations described above are sequentially coupled to transport calculations using a standard non-iterative operator splitting technique (Steefel \& MacQuarrie, 1996). The numerical solution of the model yields the spatio-temporal distributions of all chemical species and biomasses considered, as well as the distributions of the rates of all metabolic and chemical reactions, plus the chemical fluxes at the system's boundaries.

\section{The Knowledge Base (KB)}

The $\mathrm{KB}$ contains subsets of biogeochemical process libraries, including default formulations for biogeochemical reaction rates, and parameter values. Its design provides an expandable structure permitting the easy addition of new elements (subsets, variables, processes). Each default process is documented as a separate file. The file structure is always identical. It contains the set of variables involved in the specific process, the stoichiometry of the reaction, the rate (or equilibrium) equations and the parameter values used to represent the biogeochemical reaction.

Table 3 shows such a file structure using the denitrification process as an example. In this case, the rate formulation and parameter values have been obtained 
Table 3. Example of file structure included in the Knowledge Base: the denitrification process.

\begin{tabular}{|c|c|}
\hline Number of variables & 4 \\
\hline Variable names $(t)$ & $\mathrm{CH}_{2} \mathrm{O} \quad \mathrm{NO}_{3}^{-} \quad \mathrm{CO}_{2} \quad \mathrm{HCO}_{3}^{-}$ \\
\hline Stoichiometry $(2)$ & $\begin{array}{lllll}-1 & -94.4 / 106 & 13.6 / 106 & 92.4 / 106\end{array}$ \\
\hline Type of reaction & Kinetic \\
\hline Rate law & $k^{0} 10^{\alpha\left(T-T_{0}\right)} \frac{\mathrm{CH}_{2} \mathrm{O}}{\mathrm{CH}_{2} \mathrm{O}+\mathrm{K}_{\mathrm{CH}_{2} \mathrm{O}}} \frac{\mathrm{NO}_{3}^{-}}{\mathrm{NO}_{3}^{-}+\mathrm{K}_{\mathrm{NO}_{3}}} \frac{\mathrm{Kin}_{\mathrm{O}_{2}}}{\mathrm{O}_{2}+\mathrm{Kin}_{\mathrm{O}_{2}}}$ \\
\hline Number of parameters & 5 \\
\hline Parameter names $(3)$ & $\begin{array}{lllll}k^{0} & \alpha & K_{\mathrm{CH}_{2} \mathrm{O}} & K_{\mathrm{NO}_{3}^{-}} & \mathrm{Kin}_{\mathrm{O}_{2}}\end{array}$ \\
\hline Parameter values & $10^{-4} \quad(1 / 22.6) \quad 60 \quad 45 \quad 30$ \\
\hline
\end{tabular}

${ }^{(1)} \mathrm{N}_{2}$ is not explicitly computed in the model; ${ }^{(2)}$ based on Redfield ratio; ${ }^{(3)}$ units of $k$ in $\mu M . \mathrm{s}^{-1} ; K$ in $\mu \mathrm{M} ; \alpha$ in $\mathrm{K}^{-1}$.

by conducting process-oriented laboratory experiments carried out in advance of the modeling process. On this basis, the following rate equation (in mol C. $1^{-1} \cdot \mathrm{s}^{-1}$ ) has been proposed (Regnier at al., 1997):

$$
\begin{aligned}
& R_{d e n i t}=k^{0}\left(T_{0}\right) 10^{\alpha\left(T-T_{0}\right)} \frac{\mathrm{CH}_{2} \mathrm{O}}{\mathrm{CH}_{2} \mathrm{O}+\mathrm{K}_{\mathrm{CH}_{2} \mathrm{O}}} \\
& \frac{\mathrm{NO}_{3}^{-}}{\mathrm{NO}_{3}^{-}+\mathrm{K}_{\mathrm{NO}_{3}^{-}}} \frac{\mathrm{Kin}_{\mathrm{O}_{2}}}{\mathrm{O}_{2}+\mathrm{Kin}_{\mathrm{O}_{2}}}
\end{aligned}
$$

where $k^{0}\left(\mathrm{~mol} \mathrm{C} .1^{-1} \cdot \mathrm{s}^{-1}\right)$ is the rate constant at a reference temperature $T_{0}$, when there is no substrate or terminal electron acceptor limitation; $\alpha\left(\mathrm{K}^{-1}\right)$ is a parameter which defines the in-situ temperature, $\mathrm{T}$, dependence of the rate; $K_{\mathrm{CH} 2 \mathrm{O}}$ and $K_{\mathrm{NO}}\left(\mathrm{mol} . \mathrm{1}^{-1}\right)$ are the half-saturation constants with respect to the concentration of organic carbon (represented by the symbol $\mathrm{CH}_{2} \mathrm{O}$ ) and nitrate, respectively; and $\mathrm{Kin}_{\mathrm{O} 2}\left(\mathrm{~mol} \mathrm{.1} \mathrm{l}^{-1}\right)$ is a parameter which models the inhibition of the denitrification process when sufficient amount of oxygen is present in the system. As an example, Fig. 2 shows the results of process-oriented laboratory-experiments used to determine $\mathrm{K}_{\mathrm{NO} 3}$ and $\mathrm{Kin}_{\mathrm{O} 2}$, respectively.

The Knowledge Base currently assembles the information required to model the cycles of $\mathrm{C}, \mathrm{N}, \mathrm{H}, \mathrm{O}$, $\mathrm{S}, \mathrm{Fe}$, and $\mathrm{Mn}$ in redox-stratified environments. The following sets of reactions are considered: primary production, organic matter oxidation pathways, secondary redox processes (chemolithotrophic and abiotic redox reactions), aqueous complex formation, homogeneous acid dissociation reactions, non-redox mineral precipitation and dissolution, ion exchange, as well as gas-water reactions. Important goals of the
BRNS are to evaluate alternative process formulations, develop diagnostic indicators of biogeochemical pathways that can be measured in the field or in experimental set-ups, and assess the propagation of model and experimental uncertainties in predicted system behavior. Another important goal of the BRNS is to promote the transfer of knowledge be-

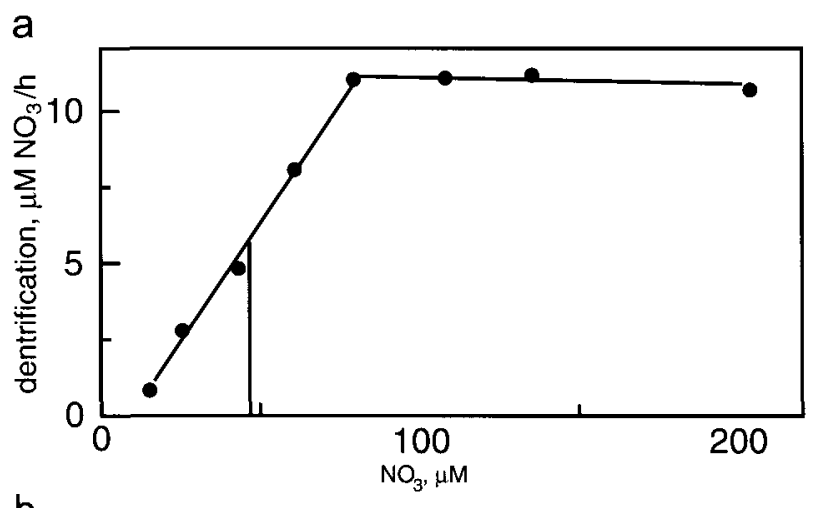

b

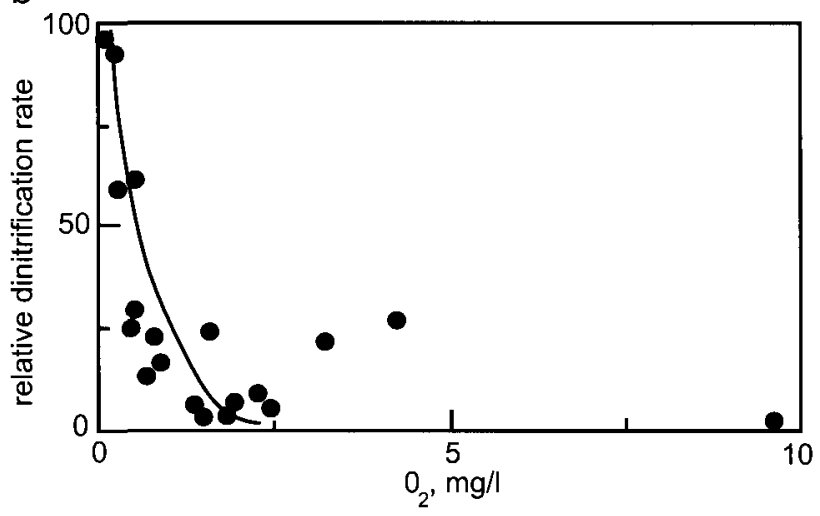

Fig. 2. Process-based laboratory experiments: determination of parameters involved in the denitrification process. (A) Relation between denitrifying activity and nitrate concentrations (from Somville, 1980) (B) Inhibition of the denitrification process by molecular oxygen (from Billen, 1976). 
tween the different subfields of reactive transport modeling, given the unifying conceptual and mathematical principles underlying all RTMs. The next section illustrates, by means of examples, the range of applicability of the proposed BRNS.

\section{Application of the BRNS to the modeling of car- bon dynamics in redox-stratified environments}

The BRNS has been applied to the modeling of carbon dynamics in three typical redox stratified environments: a continental shelf sediment, a groundwater system contaminated with organic-rich leachate (i.e. two types of porous media applications) and an eutrophic macrotidal estuary (i.e. a surface flow application). The appropriate spatial and temporal scales, transport processes, biogeochemical reactions and variables are given in Table 4. Detailed rate expressions and equilibria conditions for the various processes included in the model are given in Van Cappellen \& Wang (1996) for the sediment application; Hunter et al. (1998) for the groundwater application; and Regnier \& Steefel (1999) and Vanderborght et al. (2002) for the estuarine application. Only the results for the groundwater application are shown here.

The carbon cycle on the Earth surface is a complex combination of organic and inorganic processes acting on different time and space scales (Wollast \& Vanderborght, 1994). The carbon fluxes are simultaneously controlled by (Billen et al., 1988, Soetaert et al., 1994, Frankignoulle et al., 1995):

- primary production;

- various heterotrophic organic matter mineralization pathways;

- re-oxidation of reduced compounds;

- exchange with air;

- buffering effects of various inorganic carbon species.

Table 4. Comparison of three multi-component reactive-transport models for redox dynamics in subsurface (coastal sediment and groundwater) and surface (macrotidal estuary) environments. $1 \mathrm{D}=1$-dimensional, $\mathrm{V}=$ vertical, $\mathrm{H}=$ horizontal.

\begin{tabular}{|c|c|c|c|}
\hline & Sediment & Groundwater & Estuary \\
\hline Model: & $\begin{array}{l}\text { Transient diagenetic } \\
\text { transport model + } \\
\text { biogeochemical } \\
\text { reaction module }\end{array}$ & $\begin{array}{l}\text { Transient groundwater } \\
\text { transport model }+ \\
\text { biogeochemical reaction } \\
\text { module }\end{array}$ & $\begin{array}{l}\text { Transient hydrodynamic } \\
\text { transport model + } \\
\text { biogeochemical reaction } \\
\text { module }\end{array}$ \\
\hline Space scale: & $1 \mathrm{DV}, 35 \mathrm{~cm} ; \Delta \mathrm{z}=0.2 \mathrm{~cm}$ & $1 \mathrm{DH}, 400 \mathrm{~m} ; \Delta \mathrm{x}=4 \mathrm{~m}$ & $1 \mathrm{DH}, 150 \mathrm{~km} ; \Delta \mathrm{x}=1 \mathrm{~km}$ \\
\hline Time scale: & $200 \mathrm{yrs}, \Delta \mathrm{t}=0.001 \mathrm{yr}$ & 22 years, $\Delta \mathrm{t}=0.0005 \mathrm{yr}$ & 2 months, $\Delta t=150 \mathrm{secs}$ \\
\hline Advection: & $0.04 \mathrm{~cm} \cdot \mathrm{yr}^{-1} \quad$ (constant) & $10 \mathrm{~m} \cdot \mathrm{yr}^{-1} \quad$ (constant) & $\sim \pm 1 \mathrm{~m} . \mathrm{s}^{1} \quad$ (variable) \\
\hline Dispersion: & $\begin{array}{l}\sim 300 \mathrm{~cm}^{2} \mathrm{yr}^{-1} \text { (molecular } \\
\text { diffusion }+ \text { bioturbation } \\
3 \mathrm{~cm}^{2} \mathrm{yr}^{-1} \text { ) }\end{array}$ & $40 \mathrm{~m}^{2} \mathrm{y}^{-1}$ & $\begin{array}{l}\sim 100 \mathrm{~m}^{2} \mathrm{~s}^{-1} \text { ('macroscopic' } \\
\text { turbulent diffusion) }\end{array}$ \\
\hline $\begin{array}{l}\text { Biogeochemical } \\
\text { Processes: }\end{array}$ & $\begin{array}{l}\text { Aerobic degradation of } \\
\text { organic matter, } \\
\text { denitrification, } \\
\text { dissimilatory } \mathrm{Mn} \text { and } \mathrm{Fe} \\
\text { oxide reduction, sulfate- } \\
\text { reduction, } \\
\text { methanogenesis, } \\
\text { secondary redox reactions } \\
\text { (e.g. nitrification, Mn and } \\
\text { Fe oxide precipitation), } \\
\text { carbonic acid dissociation, } \\
\text { sulphide dissociation }\end{array}$ & $\begin{array}{l}\text { Aerobic degradation of } \\
\text { organic matter, } \\
\text { denitrification, } \\
\text { dissimilatory } \mathrm{Mn} \text { and Fe } \\
\text { oxide reduction, sulfate- } \\
\text { reduction, } \\
\text { methanogenesis, } \\
\text { secondary redox reactions } \\
\text { (e.g. nitrification, Mn and } \\
\text { Fe oxide precipitation, } \\
\text { sulphide and methane } \\
\text { oxidation) }\end{array}$ & $\begin{array}{l}\text { Aerobic degradation of } \\
\text { organic matter, } \\
\text { denitrification, primary } \\
\text { production, nitrification ( } 2 \\
\text { steps), carbonic acid } \\
\text { dissociation, water } \\
\text { dissociation, } \\
\text { ammonium/ammonia } \\
\text { equilibrium, gas transfer at } \\
\text { the water-air interface, } \\
\text { settling of SPM and } \\
\text { erosion of deposited } \\
\text { sediments }\end{array}$ \\
\hline Variables: & $\begin{array}{l}\left(\mathrm{CH}_{2} \mathrm{O}\right)_{x}(\mathrm{NH} 4)_{\mathrm{y}}(\mathrm{PO} 4)_{2} \\
\mathrm{O}_{2}, \mathrm{NH}_{4}^{+}, \mathrm{NO}_{3}, \mathrm{MnO}_{2}, \\
\mathrm{Mn}^{2+}, \mathrm{Fe}(\mathrm{OH})_{3}, \mathrm{Fe}^{2+} \\
\mathrm{SO}_{4}^{2-}, \mathrm{H}_{2} \mathrm{~S}, \mathrm{CH}_{4}, \mathrm{CO}_{2} \\
+\mathrm{H}_{2} \mathrm{CO}_{3}, \mathrm{HCO}_{3}^{-}, \mathrm{CO}_{3}{ }^{2-} \\
\mathrm{HS}^{-}, \mathrm{H}^{+}\end{array}$ & $\begin{array}{l}\left(\mathrm{CH}_{2} \mathrm{O}\right)_{x}(\mathrm{NH} 4)_{y}(\mathrm{PO} 4)_{z} \\
\text { (present as Dissolved } \\
\text { Organic Carbon; DOC), } \\
\mathrm{O}_{2}, \mathrm{NH}_{4}{ }^{+}, \mathrm{NO}_{3}^{-}, \mathrm{MnO}_{2}, \\
\mathrm{Mn}^{2+}, \mathrm{Fe}(\mathrm{OH})_{3}, \mathrm{Fe}^{2+} \\
\mathrm{SO}_{4}{ }^{2-}, \mathrm{H}_{2} \mathrm{~S}, \mathrm{CH}_{4}\end{array}$ & $\begin{array}{l}\left(\mathrm{CH}_{2} \mathrm{O}\right)_{\mathrm{x}}(\mathrm{NH} 4)_{\mathrm{y}}(\mathrm{PO} 4)_{2,}, \mathrm{O}_{2}, \\
\mathrm{NH}_{4}{ }^{+}, \mathrm{NO}_{2}^{-}, \mathrm{NO}_{3}{ }^{-}, \mathrm{NH}_{3}, \\
\mathrm{~N}_{2} \mathrm{O}, \mathrm{CO}_{2}+\mathrm{H}_{2} \mathrm{CO}_{3}, \mathrm{HCO}_{3}, \\
\mathrm{CO}_{3}{ }^{2-}, \mathrm{H}^{+}, \mathrm{OH}^{-}, \\
\text {phytoplankton, nitrifying } \\
\text { bacteria, SPM, } \mathrm{NaCl}\end{array}$ \\
\hline
\end{tabular}


Organic matter can be produced in-situ via primary production, or may enter the system through its boundaries. A metabolically diverse population of heterotrophic bacteria couples the process of organic carbon oxidation to the reduction of terminal electron acceptors (TEAs). TEAs are primarily $\mathrm{O}_{2}, \mathrm{NO}_{3}^{-}$, $\mathrm{Fe}(\mathrm{III}), \mathrm{Mn}(\mathrm{IV}), \mathrm{SO}_{4}{ }^{2-}$, and methane. Sequential utilization of these TEAs is generally assumed to occur in order of decreasing free energy yield, resulting in a stratification of microbial community structure, and chemical redox conditions (Berner, 1980; Canfield, 1993). Anaerobic decomposition of organic matter at any point in the system produces reduced inorganic species (e.g., dissolved sulphide, $\mathrm{Fe}^{2+}$, iron sulphides, $\mathrm{Mn}^{2+}$, ammonium). These species may move to another point as a result of advective and mixing processes, where they may undergo re-oxidation processes via chemolithotrophic and abiotic redox reactions. The relative intensities of these biogeochemical reactions vary significantly as a function of environmental conditions (e.g. temperature, organic and inorganic fluxes through the boundaries or material surfaces of the system).

In an estuarine environment, most of the organic carbon flux is generally mineralized via aerobic degradation $\left(\mathrm{O}_{2}\right.$ is efficiently supplied to the waters via exchange with the atmosphere), and denitrification (nitrogen loadings are important in such eutrophic environments with concentrations up to $1 \mathrm{mMN}-$ Regnier et al., 1997). In such a system, the only important inorganic reduced species produced is ammonium, which can be re-oxidized by the chemolithotrophic nitrification process in the well oxygenated portion of an estuary. The biogeochemical reaction network implemented for the estuarine case is schematized in Fig. 3.

In the diagenetic application, the sediment is located below the photic zone and has no direct contact with the atmosphere. In addition, the organic carbon supply to the sediment is relatively large on a continental shelf. These combined conditions, while preventing photosynthetic activity, trigger sulfate reduction and methano genesis as two important mineralization pathways in anoxic marine sediments. Dissimilatory iron and manganese reductions are two other common oxidants in these restricted environments. All these processes release reduced species $\left(\mathrm{Mn}^{2+}, \mathrm{Fe}^{2+}, \mathrm{H}_{2} \mathrm{~S}\right.$, $\mathrm{CO}_{2}$ ), which upon upward diffusion in the sediment column are reoxidized via secondary redox reactions.

In the groundwater application, we assume discharge of organic-rich leachate with a high reactivity to a pristine aquifer. The dissolved organic carbon (DOC) from the landfill is assumed to be the only source of organic material. After 22 years of recharge, the aquifer shows a typical redox sequence for aquifers contaminated with electron donor-rich leachates (Fig. 4A, B, C); Hunter et al., 1998; Chapelle, 2001): the concentrations of reduced species (e.g. $\mathrm{CH}_{4}, \mathrm{Fe}^{2+}, \mathrm{Mn}^{2+}$ ) are highest near the leachate source and $\mathrm{O}_{2}$ is only present downstream of the plume. Model calculations show that the DOC is entirely degraded in the first $15 \mathrm{~m}$ of the aquifer through denitrification, sulfate reduction and methano genesis (Fig. 4D). All production of dissolved $\mathrm{Fe}^{2+}$ and $\mathrm{Mn}^{2+}$ and removal of $\mathrm{O}_{2}, \mathrm{Fe}^{2+}, \mathrm{Mn}^{2+}$, $\mathrm{H}_{2} \mathrm{~S}$ and $\mathrm{CH}_{4}$ takes place through secondary redox reactions (Fig. $4 \mathrm{E}, \mathrm{F}$ ). This illustrates the potential importance of secondary redox reactions in contaminated aquifers (Hunter et al., 1988). The redox sequence is the reverse of that typically observed in sediments and pristine, electron donor-poor aquifers.

The absence of photosynthesis and gas exchange through material surfaces, and the presence of $\mathrm{Mn}$, $\mathrm{Fe}, \mathrm{SO}_{4}^{2-}$ reduction, and methano genesis explain most of the differences between the estuarine and sediment/groundwater applications. These differences in model applications are easily resolved by choosing the relevant set of variables and processes from the

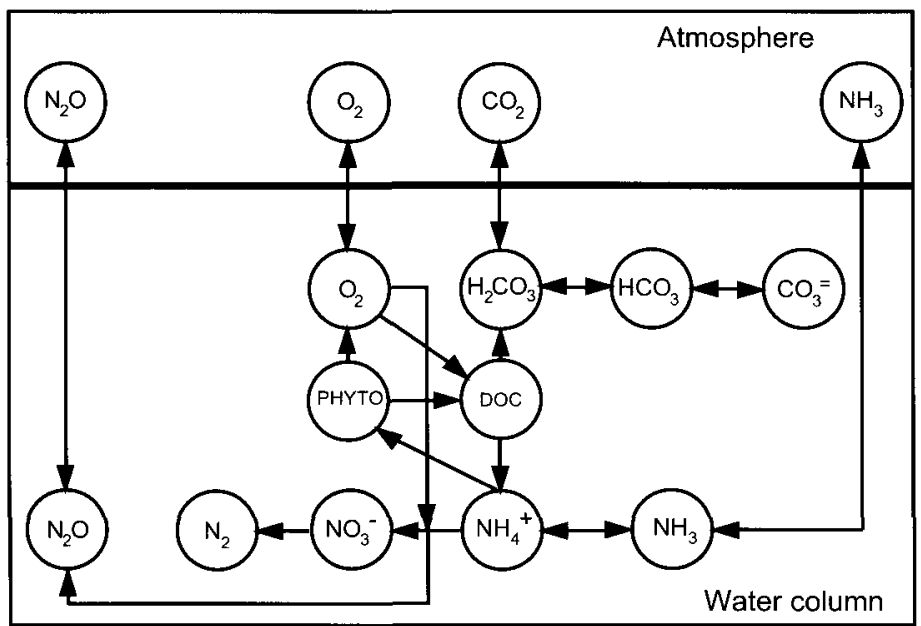

Fig. 3. Example of a complex biogeochemical reaction network: Couplings involved in the simulation of the $\mathrm{C}$ cycle and associated elements in eutrophic estuaries. 

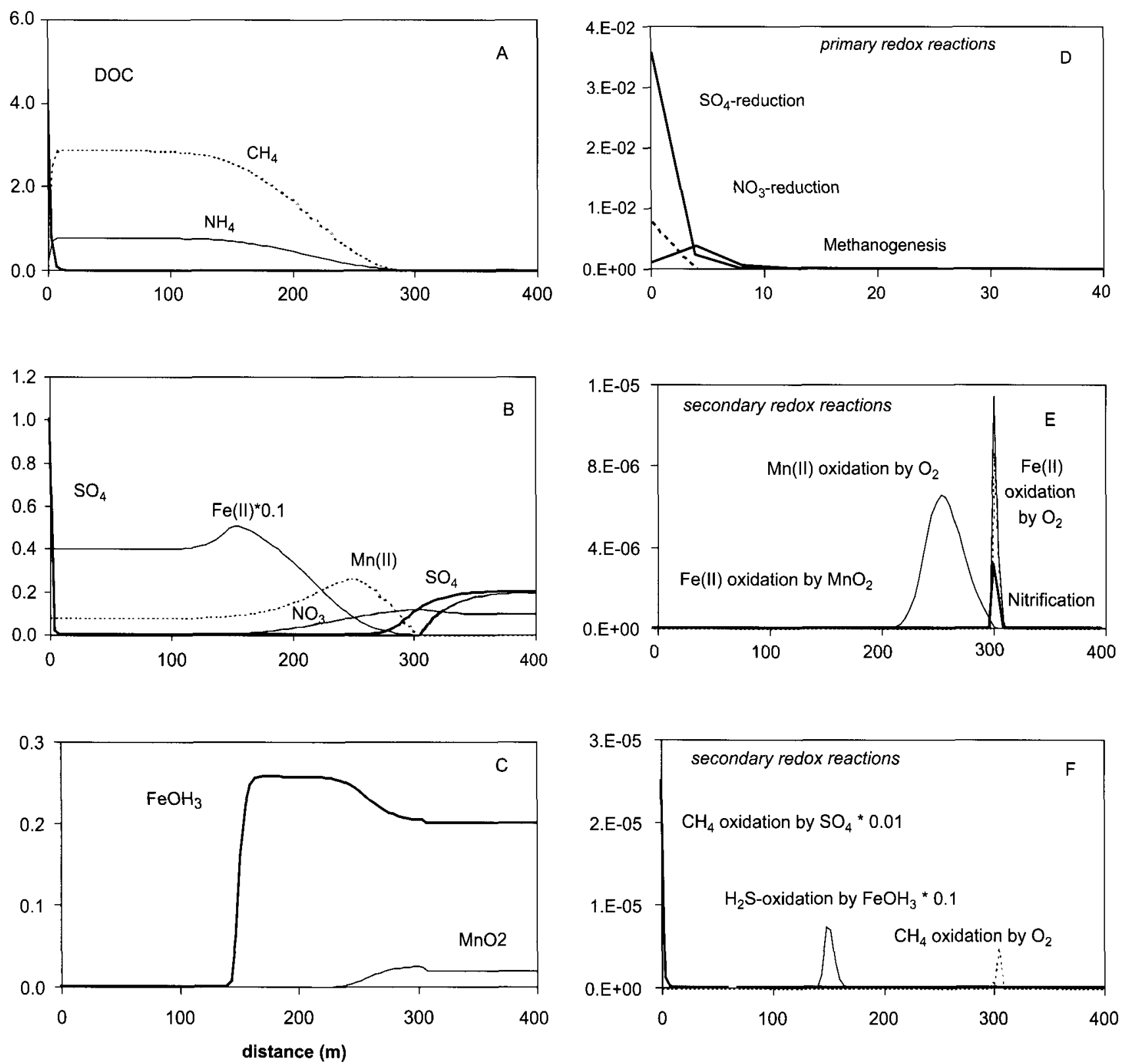

Fig. 4. Simulated concentrations of (A) DOC, $\mathrm{NH}_{4}, \mathrm{CH}_{4}$ (in $\mathrm{mM}$ ) (B) $\mathrm{SO}_{4}^{2-}, \mathrm{Fe}^{2+}, \mathrm{Mn}^{2+}, \mathrm{NO}_{3}^{-}, \mathrm{O}_{2}$ (in $\mathrm{mM}$ ) $(\mathrm{C}) \mathrm{Fe}(\mathrm{OH})_{3}$ and $\mathrm{MnO}_{2}$ and rates of (D) sulfate reduction, denitrification, methano genesis (in $\mathrm{M} \mathrm{yr}^{-1}$ ) (E) $\mathrm{Mn}^{2+}$ oxidation by $\mathrm{O}_{2}, \mathrm{Fe}^{2+}$ oxidation by $\mathrm{O}_{2}$, $\mathrm{Fe}^{2+}$ oxidation by $\mathrm{MnO}_{2}$, nitrification (in $\mathrm{M} \mathrm{yr}^{-1}$ ) (F) $\mathrm{H}_{2} \mathrm{~S}$ oxidation by $\mathrm{FeOH}_{3}, \mathrm{CH}_{4}$ oxidation by $\mathrm{O}_{2}, \mathrm{CH}_{4}$ oxidation by $\mathrm{SO}_{4}$ (in $\mathrm{M}^{-1}$ ) in an aquifer subject to 22 years of recharge with contaminated leachate with a high reactivity $\left(k=30 \mathrm{yr}^{-1}\right)$. Note the different distance scale of panel $\mathrm{D}$.

$\mathrm{KB}$. Thus, the $\mathrm{KB}$ approach to the development of biogeochemical reaction network modules facilitates the application of RTMs to a wide variety of environments facing similar geochemical problems: rivers and estuaries, coastal environments, sediments, aquifers, soils, wetlands, and geohydrological systems.

\section{Conclusions}

In this paper, we have shown that the mathematical models required to describe coupled transport and chemical reactions in many environments of the hydrosphere are fundamentally the same. Indeed, even though the levels of dynamical, spatial, and temporal approximations inherent to reactive-transport models may differ significantly, the continuum representation of mass transport relies on the same fundamental set of conservation laws. The similarity in the approach can be demonstrated by describing the mathematical foundation of three reactive-transport models applied to an estuary, coastal sediment, and a groundwater system.

An important challenge in the area of reactivetransport modeling is to develop a comprehensive set of theoretical and computational tools that allow the systematic integration of new knowledge about the 
coupled biogeochemical processes, which together, determine the cycling of elements in environmental systems. Currently, many field- and laboratory-based experiments are conducted to identify reaction pathways, quantify reaction kinetics, and elucidate the interactions between biotic and abiotic processes. The knowledge about key reaction processes involved in the biogeochemical dynamics of natural environments is therefore continuously expanding. This knowledge needs to be interfaced with the transport processes characterizing constituent movement in the various compartments of the hydrosphere. In this paper, we have proposed a Biogeochemical Reaction Network Simulator (BRNS), based on the novel concept of an evolving 'Knowledge Base' (KB). In this approach, it is no longer the model itself, but rather an easily accessible, open resource element, the KB, which contains the growing conceptual and quantitative understanding of individual biogeochemical pathways and their interactions. The proposed BRNS constitutes a particularly flexible and adaptable modeling tool for simulating the complex dynamics of biogeochemical systems in surface and sub-surface environments.

\section{References}

Berner, R.A. 1980. Early diagenesis: A theoretical approach. Princeton University Press, Princeton: $256 \mathrm{pp}$.

Billen, G. 1976. Etude ecologique des transformations de l'azote dans les sediments marins. These de l'Universite Libre de Bruxelles, Brussels, Belgium.

Billen, G., Lancelot, C., De Becker, E. \& Servais, P. 1988. Modelling microbial processes (phyto- and bacterioplankton) in the Schelde estuary, Hydrobiological Bulletin, 22: 43-55.

Boudreau, B. P., 1997. Diagenetic models and their implementation. Springer, Berlin: $414 \mathrm{pp}$.

Canfield, D.E., 1993. Organic matter oxidation in marine sediments. In: Wollast, R., Mackenzie. F.T. \& Chou, L. (eds): Interactions of $\mathrm{C}, \mathrm{N}, \mathrm{P}$ and $\mathrm{S}$ Biogeochemical Cycles and Global Change. Springer-Verlag, Berlin.

Chapelle F.H., 2001. Ground-water microbiology and Geochemistry. John Wiley \& Sons, New-York: 477 pp.

Chilakapati, A., 1995. RAFT: A Simulator for Reactive Flow and Transport of Groundwater Contaminants, Pacific Northwest Laboratory Internal Report 10636.

Ferris, M.C., Mesnier, M.P. \& More, J. J., 2000. NEOS and Condor: solving optimization problems over the Internet. ACM transactions on Mathematical software 26: 1-18.

Frankignoulle, M., Bourge, I. \& Wollast, R., 1996. Atmospheric $\mathrm{CO}_{2}$ fluxes in a highly polluted estuary (The Scheldt). Limnolology \& Oceanography 41

Hirsch, C., 1988. Numerical computation of internal and external flows, Wiley, New-York: $513 \mathrm{pp}$.

Hunter, K.S., Wang, Y. \& Van Cappellen, P., 1998. Kinetic modeling of microbially-driven redox chemistry of subsurface environ- ments: coupling transport, microbial metabolism and geochemistry. Journal of Hydrology 209: 53-80.

Lasaga, A.C., 1997. Kinetic theory in the Earth Sciences. Princeton University Press, Princeton: 811 pp.

de Marsily, G., 1986. Quantitative hydrogeology. Academic Press, New-York: 440 pp.

Nihoul, J.C.J., 1975. Modelling of Marine Systems, Elsevier Oceanography Series 10, Amsterdam: $272 \mathrm{pp}$.

Nihoul, J.C.J. \& Dienidi, S., 1991. Hierarchy and scales in marine ecohydrodynamics. Earth-Science Reviews 31:255-277.

Nihoul, J.C.J., 1993. Application of mathematical modelling to the marine environment. E. Riga. Liege.

Officer, C.B., 1976. Physical oceanography of estuaries, Wiley, New-York: 465 pp.

O'Kane, J.P., 1980. Estuarine Water Quality Management. Pitman, London: $155 \mathrm{pp}$.

Regnier, P., Wollast, R. \& Steefel, C.I., 1997. Long term fluxes of reactive species in macrotidal estuaries: Estimates from a fully transient, multi-component reaction transport model. Marine Chemistry 58: 127-145.

Regnier, P., Mouchet, A., Wollast, R. \& Ronday, F., 1998. A discussion of methods for estimating residual fluxes in strong tidal estuaries. Continental Shelf Research 18: 1543-1571.

Regnier, P. \& Steefel, C.I., 1999. A high resolution estimate of the inorganic nitrogen flux from the Scheldt estuary to the coastal North Sea during a nitrogen-limited algal bloom, Spring 1995. Geochimica \& Cosmochimica Acta 63:1359-1374.

Regnier, P., Vanderborght, J.P., Steefel, C.I. \& O'Kane, J.P., 2002. Modeling complex multi-component reactive-transport systems: Towards a simulation environment based on the concept of a Knowledge Base. Applied Mathematical Modelling, 26: 913-927.

Schwartzenbach, R.P., Gschwend, P.M. \& Imboden, D.M., 1993. Environmental organic geochemistry. Wiley Interscience, NewYork.

Soetaert, K., Herman, P.M.J. \& Kromkamp, J., 1994. Living in the twighlight: estimating net phytoplankton growth in the Westerschelde estuary (The Netherlands) by means of an ecosystem model (MOSES). Journal of Plankton Research 16: 1277-1301.

Somville, M., 1980. Etude ecophysiologique des metabolismes bacteriens dans l'estuaire de l'Escaut. These de l'Universite Libre de Bruxelles, Brussels, Belgium.

Steefel, C.I. \& MacQuarrie, K.T.B., 1996. Approaches to modelling of reactive transport in porous media. In: Reactive Transport in Porous Media. In: Lichtner, P.C., Steefel, C.I. \& Oelkers, E.H). Reviews in Mineraloly, Mineral. Soc. Amer, Washington.

Stewart, R.W., 1975 Physical Modelling. In: Modelling of Marine Systems, Vol 10. (eds. J.C.J. Nihoul) Elsevier, Amsterdam: 155167.

Van Cappellen, P. \& Wang, Y., 1996. Cycling of iron and manganese in surface sediments: a general theory for the coupled transport and reaction of carbon, oxygen, nitrogen, sulfur, iron, and manganese. American Journal of Science, 296: 197-243.

Vanderborght, J.P., Wollast, R., Loijens M. \& Regnier, P., 2002. Application of a transport-reaction model to the estimation of biogases fluxes in the Scheldt estuary. Biogeochemistry 59, 1-2: 207-237.

Wollast, R. \& Vanderborght, J-P., 1994. Aquatic carbonate systems: chemical processes in natural waters and global cycles. In: Bidoglio, G. \& Stumm, W. (eds): Chemistry of Aquatic Systems: Local and Global Perspectives ECSC, EEC, EAEC, Brussels and Luxembourg: 47-71. 